\title{
Propiedades Psicométricas de la Escala de Creencia en un Mundo Justo General y Personal en el Contexto Argentino
}

\section{Psychometric Properties of the Personal and General Belief in a Just World Scales in the Argentine Context}

\author{
Alicia Barreiro \\ Universidad de Buenos Aires \\ Consejo Nacional de Investigaciones \\ Científicas y Técnicas
}

\author{
Edgardo Etchezahar \\ Universidad de Buenos Aires \\ Consejo Nacional de Investigaciones \\ Científicas y Técnicas \\ Universidad de Lomas de Zamora
}

\author{
Vicente Prado-Gascó \\ Universidad de Valencia
}

\begin{abstract}
El objetivo del estudio fue analizar las propiedades psicométricas de las Escalas de Creencia en un Mundo Justo (CMJ) General y Personal. Por medio de análisis factorial confirmatorio se examinó la estructura interna de ambas, testeando si evalúan un mismo constructo global o si constituyen dos dimensiones diferenciadas. También se evaluó la consistencia interna de cada una, por medio de alfa de Cronbach, y sus relaciones lineales con las orientaciones religiosas. Por medio de un muestreo no probabilístico incidental, participaron 328 estudiantes universitarios de la Ciudad Autónoma de Buenos Aires, Argentina, con edades entre 18 y 40 años $(M=24,1, D T=3,88)$. El 36,6\% eran hombres el $63,4 \%$, mujeres. Los resultados del estudio sugieren propiedades psicométricas adecuadas para ambas escalas de CMJ. Además, pudo observarse que ambas escalas indagan constructos independientes, aunque relacionados. Esto último se refuerza al analizar las relaciones entre ambos tipos de CMJ y las orientaciones religiosas. Por lo tanto, este trabajo brinda herramientas válidas y confiables para la evaluación de la Creencia en un Mundo Justo Personal y General en el contexto argentino.
\end{abstract}

Palabras clave: creencia mundo justo, personal, general, orientaciones religiosas, propiedades psicométricas

\begin{abstract}
The aim of this study was to analyze the psychometric properties of the Personal and General Belief in a Just World (BJW) Scales. Through a confirmatory factor analysis, the internal structure of both scales was examined by testing if they measure the same global construct or if they constitute two different dimensions. The internal consistency of each scale was evaluated with Cronbach's alpha; also, their linear relations with religious orientations were tested. Using convenience sampling, 328 college students from the Autonomous City of Buenos Aires, Argentina, were enrolled in the study. Their ages ranged from 18 to 40 years $(M=24.1, S D=3.88) ; 36.6 \%$ were men and $63.4 \%$ were women. Results suggest appropriate psychometric properties for both BJW scales. In addition, it was observed that both scales refer to independent but related constructs. This finding is reinforeced by analyzing the relation between both types of BJW and religious orientations. Therefore, this study provides valid and reliable tools to evaluate the Personal and General BJW in Argentina.
\end{abstract}

Keywords: belief in a just world, personal, general, religious orientations, psychometric properties

Las situaciones de injusticia resultan amenazantes para los individuos, por lo tanto, para mantener una sensación de control sobre el medio las personas necesitan pensar que en la vida se tiene lo que se merece. Así, esta creencia en un mundo justo (CMJ; Lerner 1980, 1998; Lerner \& Clayton, 2011; Lerner \& Simmons

Alicia Barreiro, Facultad Latinoamericana de Ciencias Sociales-Argentina, Universidad de Buenos Aires y Consejo Nacional de Investigaciones Científicas y Técnicas (CONICET), Buenos Aires, Argentina; Edgardo Etchezahar, Centro Interdisciplinario de Investigaciones en Psicología Matemática y Experimental-CONICET, Universidad de Buenos Aires y Universidad Nacional de Lomas de Zamora, Buenos Aires, Argentina; Vicente Prado-Gascó, Departamento de Psicología Social. Facultad de Psicología, Universidad de Valencia, España.

Este trabajo ha sido posible gracias al subsidio PICT 2016-0397 "Construcción de la justicia social: representaciones sociales, prejuicio y compromiso cívico de los jóvenes” del Fondo para la Investigación Científica y Tecnológica-Agencia Nacional de Promoción Científica y Técnica, Argentina.

La correspondencia relativa a este artículo debe ser dirigida a Alicia Barreiro, FLACSO-Argentina, Tucumán 1966 (C1050AAN), Ciudad Autónoma de Buenos Aires, Argentina. E-mail: abarreiro@flacso.org.ar 
1966) responde a la necesidad de vivir en un entorno estable y predecible, donde sea posible planificar el futuro y sentirse a salvo de la injusticia. Desde que nacen los individuos estructuran su entorno físico y social apelando a propiedades estables y, de esta manera, realizan predicciones sobre el resultado de las acciones propias y ajenas o de ciertas situaciones. Por lo tanto, la ocurrencia de algo inesperado es vivida como una injusticia, dado que es algo que no tendría que haber ocurrido. De esta manera, ante una situación de injusticia, las personas reaccionan de manera automática, por pura asociación, a partir de dos patrones normativos construidos tempranamente en el desarrollo: "cosas malas le pasan a la gente mala" y "las cosas malas son causadas por malas personas" (Lerner, 1998, p. 259). Más aún, cuando no es posible identificar a un culpable por las injusticias, se justifica lo ocurrido apelando a algún tipo de intervención divina o de la naturaleza (Barreiro, 2013; Lerner, 1998; Maës, 1998).

Por otra parte, la CMJ cumple funciones sumamente relevantes para la adaptación al medio y el afrontamiento de situaciones vitales, por lo tanto, los individuos intentan protegerla cuando se ve amenazada por la existencia de injusticias (Furnham, 2003). Específicamente, la negación de las injusticias mediante la CMJ tiene dos consecuencias, una positiva y otra negativa. Por un lado, al estar relacionada directamente con actitudes autoritarias y discriminatorias, tendría efectos nocivos para la vida social en su conjunto (Barreiro, Etchezahar \& Prado-Gascó, 2014; Etchezahar, 2012; Etchezahar, Prado-Gascó, Jaume \& Brussino, 2014; Jost \& Hunyady, 2005). Por otro lado, sería salugénica para los individuos, ya que permite planificar un futuro y se relaciona directamente con estrategias de afrontamiento (Dalbert, 2001). Generalmente, las personas se piensan a sí mismas como buenas, entonces, si tienen que atravesar una situación que les genera padecimiento, la consideran como algo pasajero que tarde o temprano terminará de manera favorable. De esta manera, la CMJ permite que las personas afronten de manera favorable su propia victimización, reduciendo las posibilidades de sufrir depresión y estrés (Dalbert, 2001). Además, quienes creen que el mundo es justo invierten más en su futuro, porque confían en que sus esfuerzos serán bien recompensados (Hafer, 2000; Otto \& Dalbert, 2005). La CMJ no tendría solo una finalidad egoísta (Lerner \& Clayton, 2011), ya que se relaciona directamente con conductas pro-sociales, por cuanto es posible implementar alguna ayuda concreta para colaborar con las víctimas de injusticias (Bierhoff, Klein \& Kramp, 1991; Lerner, 1977; Lerner \& Simmons, 1966). En esta línea, los individuos con una alta CMJ piensan que las interacciones sociales tienden a ser justas y, por lo tanto, esperan ser tratados bien por los otros miembros de la sociedad (Tomaka $\&$ Blascovich, 1994). Finalmente, cabe mencionar que no se han identificado diferencias en los niveles de CMJ según el género de los individuos (Furnham, 2003).

Sin embargo, ya a fines de los 70 Lerner y Miller (1978) señalaron que la CMJ se manifiesta de forma diferente si se consideran eventos del plano personal, es decir, de la propia experiencia con el mundo, que si se trata de situaciones sociales generales. Esta diferenciación, sumada a los problemas en el análisis de la dimensionalidad del constructo (Furnham \& Procter, 1992), llevó a que a mediados de los 90 Dalbert (1999, 2001) planteara una diferenciación entre dos dimensiones de la CMJ: general (CMJG) y personal (CMJP). Mientras que la primera se refiere a la creencia en la justicia en el mundo de manera general, aunque las experiencias personales puedan ser diferentes, la segunda plantea que los eventos que han tenido lugar a lo largo de la propia vida son justos, aunque para los demás pueda no haber sido así. Por lo general, los estudios que se han ocupado de las relaciones entre ambas creencias señalan que las personas tienden a pensar que el mundo es más justo con respecto a ellos mismos que con respecto a los otros (Bègue, 2002; Calhoun \& Cann, 1994; Dalbert, 1999; Lipkus, Dalbert \& Siegler, 1996). Además, sufrir de manera personal una injusticia resulta más amenazante y es negada con mayor fuerza que cuando le ocurre a otros (Dalbert \& Yamauchi, 1994). Incluso, los individuos tienden a defender con mayor fuerza su CMJP en comparación con la CMJG (Dalbert, 1999, 2001). Por lo tanto, los resultados obtenidos por estudios previos indican que en un mismo sujeto los niveles de CMJP tienden a ser mayores que los correspondientes a la CMJG (Dalbert, 1999, 2001).

Como en las sociedades se presentan diferentes tipos de injusticias sociales y económicas, la CMJ opera contribuyendo al mantenimiento del orden social (Jost \& Hunyady, 2005) mediante la negación de las injusticias y la culpabilización de sus víctimas (e.g., Aderman, Brehm \& Katz, 1974; Lerner, 1980; Maës, 1998; Suttton \& Doglas, 2005; Wolfradt \& Dalbert, 2003). Distintos autores señalan que la CMJ es el resultado de un proceso de apropiación ideológica (Barreiro, 2008, 2013; Barreiro \& Castorina, 2006, 2015), debido a que se trata de una visión del mundo que justifica y legitima las injusticias sociales (Augoustinos, 1999; Barreiro \& Zubieta, 2011; Jost \& Hunyady, 2005). Dicho de otro modo, los hechos sociales amenazantes (como la pobreza o la discriminación) serían negados mediante la CMJ (Furnham, 2003). De esta manera, las personas evitarían sentirse expuestas a situaciones indeseables que, si bien hoy le ocurren a otro, en el futuro podrían ocurrirle a ellas (Murray, Spadafore \& Mcintosh, 2005). 
Uno de los principales constructos con los que se ha estudiado tradicionalmente la CMJ es la orientación religiosa (Lerner, 1991; Saroglou, 2003, 2010), aunque los resultados que se han informado en cuanto a sus relaciones no resultan del todo concluyentes. Específicamente, las orientaciones religiosas se definen como el proceso que controla y organiza el comportamiento de aquellos individuos que adhieren a una religión (Etchezahar \& Brussino, 2013; Etchezahar \& Simkin, 2013; Maltby, 2002; Muñoz, 2004; Paloutzian, 1996). Maltby (2002), retomando los trabajos de Allport y Ross (1967), propone las categorías de orientación extrínseca (OE) e intrínseca (OI) para distinguir a los sujetos que piensan sus prácticas religiosas de manera instrumental para alcanzar fines personales o sociales (e.g., rezar para lograr la aceptación grupal), de aquellos que las llevan a cabo como un fin en sí mismo (e.g., rezar en privado). A su vez, la OE se divide en dos factores de segundo orden asociados: la orientación extrínseca personal (en adelante OEP) y la orientación extrínseca social (en adelante OES). Mientras que la OEP remite al uso de la religión como seguridad, protección, comodidad o alivio de manera personal, la OES da cuenta del uso de la religión como un medio para alcanzar objetivos sociales. Una de las principales justificaciones para la distinción de las orientaciones religiosas ha sido el análisis de sus relaciones con diferentes concepciones de justicia (Allport \& Ross, 1967; Mavor, Macleod, Boal \& Louis, 2009). De acuerdo a los hallazgos de estudios previos (Núñez-Alarcón, MorenoJiménez \& Moral-Toranzo, 2011; Sibley \& Duckitt, 2008; Watson et al., 2003), altos niveles de OI se relacionarían con el rechazo de la idea de que las costumbres sociales puedan ser arbitrarias o que las de un grupo específico puedan ser tan buenas como las de otro grupo (Altemeyer \& Hunsberger, 2005). En este sentido, Abu-Raiya y Pargament (2011) consideran que la OI y la OEP ofrecen un marco normativo tradicional, que no se encuentra presente en los sujetos con OES que, en ocasiones, contribuye al desarrollo de la tendencia a atribuir los fenómenos a causas internas a los individuos, sin tener en cuenta elementos sociales que puedan contribuir a ellas (Altemeyer, 1981; Mavor, Louis \& Laythe, 2011).

En cuanto a las relaciones entre la CMJ y las diferentes orientaciones religiosas, se realizaron diversos estudios que indican que los sujetos con mayores niveles de CMJ tendrían una OI (Dalbert \& Katona Sallay, 1996; Furnham \& Gunter, 1984; Pargament \& Hahn, 1986; Rubin \& Peplau, 1973, 1975; Zweigenhaft, Phillips, Adams, Morse \& Horan, 1985). Asimismo, Rubin y Peplau $(1973,1975)$ identificaron una correlación directa entre los niveles de la CMJ y el nivel de religiosidad, la frecuencia con la que los sujetos asistían a la iglesia y el grado en el que los participantes de su estudio creían en un Dios activo, esto es, en algo que existe más allá de las personas y participa activamente en sus asuntos. Además, Maës (1998) señala que las personas que profesan credos afines al protestantismo presentan niveles de CMJ mayores que las personas católicas, dado que la creencia de estas últimas en que la justicia divina llegará como una recompensa más allá de la vida, es diferente a la CMJ y, por lo tanto, tiene implicaciones diferentes, como, por ejemplo, la sobrevaloración de las víctimas por creerlas merecedoras de una recompensa futura. No obstante, Hunt (2000) analizó las relaciones entre la asistencia a la iglesia en grupos de protestantes, católicos y no religiosos, concluyendo que dicha práctica no se relaciona directamente con la CMJ en ninguno de los tres grupos religiosos. Por otra parte, Pichon y Saroglou (2009) señalan que las personas con OI solo estarían dispuestas a realizar conductas de ayuda hacia las personas que no amenazan su sistema de valores. Sus resultados indican que la CMJ tendría un rol mediador entre las conductas pro-sociales y la religión, porque operaría culpabilizando a las víctimas de sus padecimientos. A partir de tales estudios y de las discusiones en torno a la dimensionalidad de la CMJ, es necesario complejizar los análisis realizados, dado que los resultados obtenidos no son concluyentes, tal como afirman diferentes investigadores (e.g., Benson, 1992; Crozier \& Joseph, 1997; Hunt, 2000; Maës, 1998; Pichon \& Saroglou, 2009). En este sentido, es posible avanzar en el análisis de las relaciones entre la CMJ y la religiosidad, al considerar, por un lado, las diferentes OE (OES y OEP), además de la OI, y, por otro, poder discriminar entre la CMJP y la CMJG para el análisis de las relaciones entre ellas.

El objetivo principal de este trabajo fue analizar las propiedades psicométricas de las escalas de CMJP y la CMJG, con la finalidad de contar con instrumentos adaptados al contexto hispanoparlante para su evaluación. De esta manera también se esperaba contribuir al análisis de ambas dimensiones de la CMJ, aportando datos sobre sus relaciones en Buenos Aires. De acuerdo con los estudios previos, se esperaba una correlación moderada entre ambas dimensiones de la CMJ, con niveles más altos de CMJP en comparación con la CMJG. Para cumplimentar tales objetivos, en primer lugar, se examinó la estructura interna de ambas escalas para evaluar los dos tipos de CMJ (Dalbert, 1999, 2001), dado que no se cuenta con adaptaciones al contexto local. En segundo lugar, se analizaron las relaciones entre ambos tipos de CMJ y las orientaciones religiosas. Debido a que la CMJ requiere un alto nivel de locus de control interno (Barreiro \& Zubieta, 2011), 
se esperaba que ambos tipos de creencias se relacionen directamente con la OI. Asimismo, se esperaba que la OES se relacione en mayor medida con la CMJG, mientras que la OEP lo haga con la CMJP.

\section{Método}

\section{Diseño}

Se trabajó con un diseño de tipo instrumental (Montero García-Celay \& León, 2007), dado que el propósito principal del estudio fue analizar las propiedades psicométricas de dos evaluaciones de la creencia en un mundo justo (CMJP y CMJG) y dar cuenta de si ambas evalúan un mismo constructo global o son dos dimensiones diferentes del mismo fenómeno.

Este estudio forma parte de un proyecto más amplio, por lo tanto, ya se han realizado publicaciones con la misma muestra en las que se analizaron otras variables (Barreiro et al., 2014).

\section{Participantes}

Participaron del estudio 328 estudiantes universitarios residentes en la Ciudad Autónoma de Buenos Aires, con edades entre los 18 y los 40 años $(M=24,1 ; D T=3,88)$. El tipo de muestreo utilizado fue no probabilístico incidental. El $36,6 \%$ eran hombres $(n=120)$ y el $63,4 \%$, mujeres $(n=208)$. El $61,28 \%$ provenía de la carrera de Psicología de la Facultad de Psicología de la Universidad de Buenos Aires y el 38,72\% restante de la carrera de Sociología de la Facultad de Ciencias Sociales de la misma universidad. Además, el 5,79\% $(n=19)$ se autoposicionó como perteneciente a clase social media-baja, el $83,23 \%(n=273)$, a clase media y el $10,98 \%(n=36)$, a clase media-alta.

\section{Instrumentos}

Los datos fueron recolectados a través de un instrumento autoadministrable compuesto por las siguientes escalas:

Escala de Creencia General en un Mundo Justo. Para evaluar el constructo se realizó una adaptación de la escala original construida por Dalbert (1999; Dalbert \& Sallay, 2004), compuesta por seis ítems (ver Tabla 1). El formato de respuesta de tipo Likert fue de cinco anclajes, desde $1=$ Totalmente en desacuerdo a $5=$ Totalmente de acuerdo. Mayores puntuaciones indican mayores niveles de CMJ. Las propiedades psicométricas de la escala original unidimensional de Dalbert (1999) resultaron adecuadas $(\mathrm{CFI}=0,93 ;$ RMSEA $=0,042 ; \alpha$ de Cronbach $=0,83)$.

Escala de Creencia Personal en un Mundo Justo (Dalbert, 1999). Se realizó una adaptación de la escala original, la cual estuvo compuesta por siete ítems (ver Tabla 1) que en su conjunto evalúan la creencia en que los eventos que suceden en la vida de un individuo son justos. El formato de respuesta de tipo Likert fue de cinco anclajes, siendo $1=$ Totalmente en desacuerdo y $5=$ Totalmente de acuerdo. Mayores puntuaciones indican mayores niveles de CMJ. La escala demostró contar con propiedades psicométricas adecuadas, tanto en su construcción original (Dalbert, 1999; CFI =0,95; RMSEA $=0,034$; $a$ de Cronbach $=0,86$ ), como en sucesivas evaluaciones en diferentes contextos (ver Dalbert \& Stoeber, 2006; 0,74< $\alpha<0,91$ ).

Para la adaptación de estos dos instrumentos se siguieron las recomendaciones de la International Test Commission (Muñiz, Elosua \& Hamleton, 2013). Las escalas de CMJG y CMJP fueron traducidas al español y, posteriormente, se realizó una retro-traducción al inglés por dos traductores nativos. Posteriormente, se compararon las escalas originales con las resultantes del proceso de retrotraducción, hallando una concordancia entre ambas versiones de las escalas. Además de los ítems originales de cada una de las escalas, se incluyeron ítems extra (cinco en cada una) con la finalidad de poder reemplazar alguno de los originales, si es que sus propiedades psicométricas no resultaban adecuadas, aunque finalmente esto no resulto necesario, debido a que todos los ítems originales presentaron estadísticos descriptivos adecuados, así como también aportaron a la consistencia interna y validez de constructo de cada variable.

Escala de Orientación Religiosa (I-E Age Universal 12). Se utilizó una versión de la escala Age Universal I-E-12 (Maltby, 2002) adaptada y validada al contexto local (Simkin \& Etchezahar, 2013). La misma se compone de 12 ítems, seis de los cuales evalúan la OI (e.g., "Me esfuerzo por vivir mi vida acorde a mis creencias religiosas"), tres, la OEP (e.g., "Rezo principalmente para conseguir alivio y protección”) y tres, 
la OES (e.g., "Voy a la iglesia principalmente para pasar tiempo con mis amigos"). Se utilizó un formato de respuesta tipo Likert con cinco opciones de respuesta, que van desde $1=$ Totalmente de acuerdo a $5=$ Totalmente en desacuerdo. Mayores puntuaciones indican una mayor orientación religiosa. Las propiedades psicométricas de la escala en su validación al contexto argentino (Simkin \& Etchezahar, 2013) resultaron adecuadas, tanto en su consistencia interna $(0,79<\alpha<0,88)$ como en su validez de constructo, $\mathrm{S}-\mathrm{B} \chi^{2}(51)=89,76, p<0,001, \mathrm{NFI}=0,97, \mathrm{CFI}=0,97, \mathrm{IFI}=0,97, \mathrm{RMSEA}=0,057$.

Variables sociodemográficas. Se desarrolló un cuestionario ad-hoc para recabar información acerca del sexo y la edad de los participantes.

\section{Procedimiento}

Los individuos que participaron en esta investigación lo hicieron de manera voluntaria y anónima, luego de firmar un consentimiento informado. Además, se les informó que los datos relevados serían utilizados con fines exclusivamente académico-científicos, bajo la Ley Nacional 25.326 de protección de datos personales. La administración del cuestionario se realizó en un espacio destinado para tal fin en la universidad, por los autores, en un único encuentro con cada sujeto con una duración de aproximadamente 30 minutos.

\section{Análisis de Datos}

Los análisis estadísticos fueron realizados mediante el paquete estadístico SPSS 20.0 y el EQS 6.1. En primer lugar, se realizaron los análisis descriptivos de cada ítem (media, desviación típica correlación ítemtotal y alfa de Cronbach si se elimina el elemento). A continuación, se analizó la consistencia interna, a través de a de Cronbach, y, luego la validez de constructo de ambas escalas de CMJ. Se realizaron dos análisis factoriales confirmatorios (AFC) para corroborar si el modelo de dos dimensiones sin correlacionar o de dos dimensiones correlacionadas se ajustaba mejor a los datos obtenidos con respecto a las CMJG y CMJP. Para los AFC se utilizó como método de estimación el de máxima verosimilitud (ML) y la corrección de los datos no normales (Satorra \& Bentler, 2001) a través de la estimación robusta de Satorra-Bentler (S-B). Siguiendo las sugerencias de $\mathrm{Hu}$, Bentler y Kano (1992), para el análisis del ajuste de los modelos testeados se consideró la distribución de $\chi^{2}$ y sus grados de libertad (con estimación ML), la corrección S-B $\chi^{2}$ y sus grados de libertad (con estimación robusta) y la razón entre $\mathrm{S}-\mathrm{B}$ y $\chi^{2}\left(\mathrm{~S}-\mathrm{B} / \chi^{2}\right)$, junto con sus grados de libertad, siendo aceptables los valores inferiores a cuatro (Browne \& Cudeck, 1993). Se calcularon, además, el índice de ajuste no normalizado (NNFI), el índice de ajuste comparativo (CFI) y el índice de ajuste incremental de Bollen (IFI), siendo indicadores de un buen ajuste los valores superiores a 0,90 (MacCallum \& Austin, 2000). Además, se consideró el error cuadrático medio de aproximación (RMSEA), siendo los valores inferiores a 0,08 considerados como adecuados (Browne \& Cudeck, 1993). Finalmente, se calcularon las correlaciones lineales de Pearson entre ambas formas de la CMJ y las orientaciones religiosas.

\section{Resultados}

\section{Análisis de Ítems y Consistencia Interna}

En la Tabla 1 se presenta, para cada ítem de las escalas CMJG y CMJP, la media, desviación típica, correlación ítem-total y alfa de Cronbach si se elimina el elemento.

En la Tabla 1 puede observarse que todos los ítems presentan estadísticos descriptivos adecuados (Hair, Black, Babin, Anderson \& Tatham, 2006). Las correlaciones ítem-total indican que todos los ítems contribuyen adecuadamente al total de la escala $(0,42 \leq r \leq 0,71)$.

Los resultaron indicaron niveles de consistencia interna adecuados de ambas escalas de CMJ (CMJG: $\alpha=0,74$; CMJP: $\alpha=0,85$ ), no mejorando al eliminar ningún ítem de la escala (Tabla 1). Además, el valor medio de la CMJG fue menor $(M=2,58, D T=1,18)$ que el de la CMJP $(M=3,05, D T=1,04)$.

\section{Análisis de Validez de Constructo}

En la Tabla 2 se presentan los resultados del AFC. Como puede apreciarse, el modelo de CMJ de dos dimensiones correlacionadas presenta mejor ajuste que el modelo de dos dimensiones no correlacionadas, $\Delta$ S-B $\chi^{2}<5$, NNFI, CFI y IFI $>0,90$ y RMSEA $\leq 0,06$. Por lo tanto, es posible considerarlas como dos dimensiones diferentes aunque relacionadas. 
Tabla 1

Análisis Descriptivo de los Ítems de las Escalas de CMJG y CMJP

\begin{tabular}{|c|c|c|c|c|}
\hline Escala e ítem & $M$ & $D T$ & $r_{\mathrm{jx}}$ & a.-x \\
\hline \multicolumn{5}{|l|}{ Creencia en un Mundo Justo General $(\alpha=0,74)$} \\
\hline $\begin{array}{l}\text { CMJG 1. Tengo confianza en que la justicia siempre prevalece sobre la } \\
\text { injusticia }\end{array}$ & 2,18 & 1,22 & 0,52 & 0,70 \\
\hline $\begin{array}{l}\text { CMJG 2. Creo firmemente que las injusticias en todos los ámbitos de } \\
\text { la vida (por ejemplo, profesional, familiar, político) son la } \\
\text { excepción y no la regla }\end{array}$ & 2,59 & 1,09 & 0,45 & 0,72 \\
\hline $\begin{array}{l}\text { CMJG 3. Pienso que la gente trata de ser justa cuando toma decisiones } \\
\text { importantes }\end{array}$ & 3,08 & 1,15 & 0,42 & 0,73 \\
\hline $\begin{array}{l}\text { CMJG 4. Estoy convencido que a largo plazo la gente será compensada } \\
\text { por las injusticias }\end{array}$ & 2,93 & 1,30 & 0,49 & 0,71 \\
\hline CMJG 5. Creo que, en general, la gente obtiene lo que merece & 2,67 & 1,27 & 0,51 & 0,70 \\
\hline CMJG 6. Básicamente pienso que el mundo es un lugar justo & 2,06 & 1,06 & 0,54 & 0,70 \\
\hline \multicolumn{5}{|l|}{ Creencia en un Mundo Justo Personal $(\alpha=0,85)$} \\
\hline CMJP 1. Creo que, en general, merezco lo que me pasa & 2,84 & 1,17 & 0,58 & 0,84 \\
\hline CMJP 2. Por lo general, soy tratado justamente & 3,25 & 0,99 & 0,60 & 0,84 \\
\hline CMJP 3. Creo que generalmente obtengo lo que merezco & 3,15 & 1,02 & 0,67 & 0,83 \\
\hline CMJP 4. En general los eventos en mi vida son justos & 3,13 & 1,01 & 0,71 & 0,82 \\
\hline CMJP 5. En mi vida la injusticia es la excepción y no la regla & 3,00 & 1,09 & 0,50 & 0,85 \\
\hline $\begin{array}{l}\text { CMJP 6. Creo que la mayoría de las cosas que me pasan en la vida son } \\
\text { justas }\end{array}$ & 3,03 & 1,06 & 0,71 & 0,82 \\
\hline $\begin{array}{l}\text { CMJP 7. Pienso que las decisiones importantes que se toman con } \\
\text { respecto a mi generalmente son justas }\end{array}$ & 3,00 & 0,96 & 0,60 & 0,84 \\
\hline
\end{tabular}

Tabla 2

Análisis Factorial Confirmatorio de la Escala de CMJ

\begin{tabular}{cccccccc}
\hline & $\chi^{2}(g l)$ & $S-B \chi^{2}(g l)$ & $\Delta S-B \chi^{2}$ & NNFI & CFI & IFI & RMSEA \\
\hline CMJ (sin corr) & $253,67(65)$ & $205,52(65)$ & 3,16 & 0,84 & 0,87 & 0,87 & 0,08 \\
CMJ (dos corr) & $144,26(64)$ & $113,47(64)$ & 1,77 & 0,92 & 0,94 & 0,94 & 0,06 \\
\hline
\end{tabular}

Nota. CMJ (sin corr): Modelo de dos dimensiones sin correlacionar; CMJ (dos corr): Modelo de dos dimensiones correlacionadas.

\section{Relaciones entra Ambas CMJ y las Orientaciones Religiosas}

En la Tabla 3 se presentan las correlacionesentre ambas escalas, así como sus relaciones con las orientaciones religiosas.

De manera coherente con los resultados reportados con respecto al AFC, se identificó una relación moderada directa entre ambos tipos de CMJ $(r=0,56, p<0,01)$. Con respecto a las correlaciones entre las CMJ y las orientaciones religiosas, en la Tabla 3 puede observarse que ambas se relacionan de forma directa con la OI y con la OEP. Además, cabe señalar que no se hallaron relaciones entre la OES y las dos CMJ. 
Tabla 3

Correlaciones entre las Escalas de CMJP y CMJG y las Orientaciones Religiosas

\begin{tabular}{|c|c|c|c|c|c|c|}
\hline & $a$ & 1 & 2 & 3 & 4 & 5 \\
\hline 1. CMJP & 0,85 & - & & & & \\
\hline 2. CMJG & 0,74 & $0,56^{*}$ & - & & & \\
\hline 3. OI & 0,88 & $0,18^{*}$ & $0,29^{*}$ & - & & \\
\hline 4. OES & 0,79 & 0,02 & 0,08 & $0,54^{*}$ & & \\
\hline 5. OEP & 0,81 & $0,20^{*}$ & $0,32^{*}$ & $0,76^{*}$ & $0,43^{*}$ & - \\
\hline
\end{tabular}

\section{Discusión}

En base a los resultados obtenidos, es posible afirmar que la CMJG y la CMJP constituyen dos dimensiones independientes aunque relacionadas, no siendo posible interpretarlas de forma unidimensional. Tanto los estadísticos descriptivos para los seis ítems de la escala de CMJG como los correspondientes a los siete ítems de la escala CMJP resultaron adecuados, así como también los indicadores de validez y consistencia interna de ambas. Estos hallazgos siguen la línea de lo propuesto por Furnham y Procter (1992) con respecto a la necesidad de abandonar la unidimensionalidad que hasta el momento suponían las evaluaciones de la CMJ. Asimismo, brindan evidencia a favor de la propuesta de Dalbert $(1999,2001)$ con respecto a la diferenciación de dos formas de la CMJ (general y personal). En este sentido, se considera necesario diferenciar la creencia en la existencia de justicia en el mundo de manera general de la creencia referida a la justicia de los eventos que tuvieron lugar a lo largo de la propia vida. Asimismo, la puntuación media obtenida por los participantes de este estudio en la CMJP es mayor, en comparación con la que obtienen en la CMJG. Estos resultados son consistentes con los estudios previos que evaluaron las relaciones entre ambos tipos de CMJ (Bègue, 2002; Calhoun \& Cann, 1994; Dalbert, 1999, 2001; Lipkus et al., 1996).

Con respecto a las relaciones entre las orientaciones religiosas y los diferentes tipos de CMJ, por una parte, se observó que la OI se relaciona de forma significativa y directa tanto con la CMJG como con la CMJP. Estos resultados son coherentes con los hallazgos de estudios previos (Dalbert \& Katona-Sallay, 1996; Furnham \& Gunter, 1984; Pargament \& Hahn, 1986) y pueden interpretarse teniendo en cuenta que la CMJ implica un locus de control interno (Barreiro \& Zubieta, 2011). Dicho de otro modo, tanto la CMJP como la CMJG plantean que lo que se consiga en la vida depende de las acciones de cada persona, es decir, que estas tienen el control sobre sus vidas. En este sentido, la OI refiere a las creencias y prácticas religiosas que se realizan por propia decisión, sin considerar la aquiescencia de los otros miembros del propio grupo (Maltby, 2002). Por otra parte, con respecto a la OE, los resultados dan cuenta de relaciones directas entre ambos tipos de CMJ y la OEP; sin embargo, no se han hallado relaciones con la OES. Es posible considerar que tales resultados se deban a que, según Abu-Raiya y Pargament (2011), tanto la OI como la OEP ofrecen un marco normativo tradicional, el cual no se encuentra presente en las personas con altos niveles de OES.

Una de las principales implicancias a futuro que se propuso en este trabajo fue realizar una contribución al desarrollo de instrumentos válidos y confiables para la evaluación de las CMJP y CMJG en un contexto hispanoparlante. Además, este estudio tenía como propósito analizar las posibles relaciones entre ambas CMJ y las orientaciones religiosas, dado que se encuentran escasos antecedentes en general y particularmente a nivel local.

No obstante, se considera necesario que los futuros trabajos que indaguen este constructo consideren dos aspectos que se presentan como limitaciones a este estudio.

En primer lugar, es necesario continuar evaluando las relaciones entre los diferentes tipos de CMJ en muestras que incluyan sujetos de otros estamentos sociales, puesto que este trabajo, al igual que los realizados previamente por otros investigadores, analizó los constructos considerados en una población relativamente homogénea, compuesta por estudiantes universitarios.

En segundo lugar, se recomienda que se continúe avanzando en el estudio de las relaciones entre las creencias religiosas y la CMJ —incluyendo sus distintos tipos-, considerando diferentes religiones y diversos modos de operacionalizarlas, dado que los resultados obtenidos hasta el momento aún no resultan concluyentes. 
BARREIRO, ETCHEZAHAR Y PRADO-GASCÓ

\section{Referencias}

Abu-Raiya, H. \& Pargament, K. I. (2011). Empirically based psychology of Islam: Summary and critique of the literature. Mental Health, Religion \& Culture, 14, 93-115. https://doi.org/10.1080/13674670903426482

Aderman, D., Brehm, S. S. \& Katz, L. B. (1974). Empathic observation of an innocent victim: The just world revisited. Journal of Personality and Social Psychology, 29, 342-347. https://doi.org/10.1037/h0036001

Allport, G. W. \& Ross, J. M. (1967). Personal religious orientation and prejudice. Journal of Personality and Social Psychology, 5, 432-443. https://doi.org/10.1037/h0021212

Altemeyer, B. (1981). Right-wing authoritarianism. Winnipeg, Canadá: University of Manitoba Press.

Altemeyer, B. \& Hunsberger, B. (2005). Fundamentalism and authoritarianism. En R. F. Paloutzian \& C. L. Park (Eds.), Handbook of the psychology of religion and spirituality (pp. 378-393). New York, NY: Guilford Press.

Augoustinos, M. (1999). Ideology, false consciousness and psychology. Theory \& Psychology, 9(3), 295-312. https://doi.org/10.1177/0959354399093002

Barreiro, A. V. (2008). El desarrollo de la creencia en un mundo justo: relaciones entre la construcción individual del conocimiento y los saberes producidos colectivamente. Estudios de Psicología, 29, 289-299. https://doi.org/10.1174/021093908786145403

Barreiro, A. (2013). The appropriation process of the belief in a just world. Integrative Psychological \& Behavioral Sciences, 47, $431-449$. https://doi.org/10.1007/s12124-013-9246-y

Barreiro, A. \& Castorina, J. A. (2006). Dos perspectivas sobre la creencia en la justicia del mundo: naturalismo versus legitimación ideológica. Espacios en Blanco, 16, 97-121.

Barreiro, A. \& Castorina, J. A. (2015). La creencia en un mundo justo como trasfondo ideológico de la representación social de la justicia. Revista Colombiana de Psicología, 24, 331-345. https://doi.org/10.15446/rcp.v24n2.44294

Barreiro, A., Etchezahar, E. \& Prado-Gascó, V. (2014). Creencia global en un mundo justo: validación de la escala de Lipkus en estudiantes universitarios de la Ciudad de Buenos Aires. Interdisciplinaria, 31, 57-71. https://doi.org/10.16888/interd.2014.31.1.4

Barreiro, A. \& Zubieta, E. (2011). Parsimonia cognitiva y legitimación de las injusticias sociales: la creencia en el mundo justo y el fatalismo latinoamericano. Anuario de Investigaciones, 18, 219-226.

Bègue, L. (2002). Beliefs in justice and faith in people: Just world, religiosity and interpersonal trust. Personality and Individual Differences, 32, 375-382. https://doi.org/10.1016/s0191-8869(00)00224-5

Benson, D. E. (1992). Why do people believe in a just world? Testing explanations. Sociological Spectrum, 12, 73-104. https://doi.org/10.1080/02732173.1992.9981988

Bierhoff, H. W., Klein, R. \& Kramp, P. (1991). Evidence for the altruistic personality from data on accident research. Journal of Personality, 59, 263-280. https://doi.org/10.1111/j.1467-6494.1991.tb00776.x

Browne, M. W. \& Cudeck, R. (1993). Alternative ways of assessing model fit. En K. A. Bollen \& J. S. Long (Eds.), Testing structural equation models (pp. 136-162). Newbury Park, CA: SAGE.

Calhoun, L. G. \& Cann, A. (1994). Differences in assumptions about a just world: Ethnicity and point of view. The Journal of Social Psychology, 134, 765-770. https://doi.org/10.1080/00224545.1994.9923011

Crozier, S. \& Joseph, S. (1997). Religiosity and sphere-specific just world beliefs in 16- to 18-year-olds. The Journal of Social Psychology, 137, 510-513. https://doi.org/10.1080/00224549709595468

Dalbert, C. (1999). The world is more just for me than generally: About the Personal Belief in a Just World Scale's validity. Social Justice Research, 12, 79-98. https://doi.org/10.1023/A:1022091609047

Dalbert, C. (2001). The justice motive as personal resource: Dealing with challenges and critical life events. New York, NY: Springer.

Dalbert, C. \& Katona-Sallay, H. (1996). "The belief in a just world" construct in Hungary. Journal of Cross-Cultural Psychology, 27, 293-314. https://doi.org/10.1177/0022022196273003

Dalbert, C. \& Yamauchi, L. A. (1994). Belief in a just world and attitudes toward immigrants and foreign workers: A cultural comparison between Hawaii and Germany. Journal of Applied Social Psychology, 24(18), 1612-1626. https://doi.org/10.1111/j.1559-1816.1994.tb01565.x

Dalbert, C. \& Sallay, H. (2004). Developmental trajectories and developmental functions of the belief in a just world: Some concluding remarks. En C. Dalbert \& H. Sallay (Eds.), The justice motive in adolescence and young adulthood: Origins and consequences (pp. 248-262). New York, NY: Routledge.

Dalbert, C. \& Stoeber, J. (2006). The personal belief in a just world and domain-specific beliefs about justice at school and in the family: A longitudinal study with adolescents. International Journal of Behavioral Development, 30, 200-207. https://doi.org/10.1177/0165025406063638

Etchezahar, E. (2012). Las dimensiones del autoritarismo: análisis de la escala de autoritarismo del ala de derechas (RWA) en una muestra de estudiantes universitarios de la Ciudad de Buenos Aires. Psicologia Política, Associação Brasileira de Psicologia Política, 12, 591-603.

Etchezahar, E. \& Brussino, S. (2013). Psychological perspectives in the study of authoritarianism. Journal of Alternative Perspectives in the Social Sciences, 5, 495-521.

Etchezahar, E. \& Simkin, H. (2013). Religiosidad, espiritualidad y escepticismo: la mediación del autoritarismo. Subjetividad y Procesos Cognitivos, 17(2), 48-58.

Etchezahar, E., Prado-Gascó, V., Jaume, L. \& Brussino, S. (2014). Validación argentina de la escala de Orientación a la Dominancia Social (SDO). Revista Latinoamericana de Psicología, 46, 35-43. https://doi.org/10.1016/S0120-0534(14)70004-4

Furnham, A. (2003). Belief in a just world: Research progress over the past decade. Personality and Individual Differences, $34,795-817$. https://doi.org/10.1016/S0191-8869(02)00072-7

Furnham, A. \& Gunter, B. (1984). Just-world beliefs and attitudes towards the poor. British Journal of Social Psychology, 23, 265-269. https://doi.org/10.1111/j.2044-8309.1984.tb00637.x

Furnham, A. \& Procter, E. (1992). Sphere-specific just world beliefs and attitudes to AIDS. Human Relations, 45, 265-280. https://doi.org/10.1177/001872679204500303

Hafer, C. L. (200). Investment in long-term goals and commitment to just means drive the need to believe in a just world. Personality and Social Psychology Bulletin, 26, 1059-1073. https://doi.org/10.1177/01461672002611004

Hair Jr., J. F., Black, W. C., Babin, B. J., Anderson, R. E. \& Tatham, R. L. (2006). Multivariate data analysis (6a ed.). Upper Saddle River, NJ: Pearson.

Hu, L. -T., Bentler, P. M. \& Kano, Y. (1992). Can test statistics in covariance structure analysis be trusted? Psychological Bulletin, 112, 351-362. https://doi.org/10.1037/0033-2909.112.2.351 
Hunt, M. O. (2000). Status, religion, and the "belief in a just world": Comparing African Americans, Latinos and whites. Social Science Quarterly, 81, 325-343.

Jost, J. T. \& Hunyady, O. (2005). Antecedents and consequences of system-justifying ideologies. Current Directions in Psychological Science, 14, 260-265. https://doi.org/10.1111/j.0963-7214.2005.00377.x

Lerner, M. J. (1977). The justice motive: Some hypotheses as to its origins and forms. Journal of Personality, 45, 1-52. https://doi.org/10.1111/j.1467-6494.1977.tb00591.x

Lerner, M. J. (1980). The belief in a just world: A fundamental delusion. New York, NY: Plenum Press. https://doi.org/10.1007/978-14899-0448-5

Lerner, M. J. (1991). The belief in a just world and the 'heroic motive': Searching for 'constants' in the psychology of religious ideology. The International Journal for the Psychology of Religion, 1, 27-32. https://doi.org/10.1207/s15327582ijpr0101_4

Lerner, M. J. (1998). The two forms of belief in a just world: Some thoughts on why and how people care about justice. En L. Montada \& M. J. Lerner (Eds.), Responses to victimizations and belief in a just world (pp. 247-269). New York, NY: Plenum Press.

Lerner, M. J. \& Clayton, S. (2011). Justice and self-interest: Two fundamental motives. New York, NY: Cambridge University Press.

Lerner, M. J. \& Miller, D. T. (1978). Just world research and the attribution process: Looking back and ahead. Psychological Bulletin, 85, 1030-1051. https://doi.org/10.1037/0033-2909.85.5.1030

Lerner, M. J. \& Simmons, C. H. (1966). Observer's reaction to the "innocent victim": Compassion or rejection? Journal of Personality and Social Psychology, 4, 203-210. https://doi.org/10.1037/h0023562

Lipkus, I. M., Dalbert, C. \& Siegler, I. C. (1996). The importance of distinguishing the belief in a just world for self versus for others: Implications for psychological well-being. Personality and Social Psychology Bulletin, 22, 666-677. https://doi.org/10.1177/0146167296227002

Maës, J. (1998). Immanent justice and ultimate justice: Two ways of believing in justice. En L. Montada \& M. J. Lerner (Eds.), Responses to victimizations and belief in a just world (pp. 9-40). New York, NY: Plenum Press.

MacCallum, R. C. \& Austin, J. T. (2000). Applications of structural equation modeling in psychological research. Annual Review of Psychology, 51, 201-226. https://doi.org/10.1146/annurev.psych.51.1.201

Maltby, J. (2002). The Age Universal I-E Scale-12 and orientation toward religion: Confirmatory factor analysis. The Journal of Psychology, 136, 555-560. https://doi.org/10.1080/00223980209605550

Mavor, K. I., Louis, W. R. \& Laythe, B. (2011). Religion, prejudice, and authoritarianism: Is RWA a boon or bane to the psychology of religion? Journal for the Scientific Study of Religion, 50, 22-43. https://doi.org/10.1111/j.1468-5906.2010.01550.x.

Mavor, K. I., Macleod, C. J., Boal, M. J. \& Louis, W. R. (2009). Right-wing authoritarianism, fundamentalism and prejudice revisited: Removing suppression and statistical artefact. Personality and Individual Differences, 46, 592-597. https://doi.org/10.1016/j.paid.2008.12.016

Montero García-Celay, I. \& León, O. G. (2007). A guide for naming research studies in psychology. International Journal of Clinical and Health Psychology, 7, 847-862.

Muñiz, J., Elosua, P. \& Hambleton, R. K. (2013). Directrices para la traducción y adaptación de los tests: segunda edición. Psicothema, 25, 151-157. https://doi.org/10.7334/psicothema2013.24

Muñoz, A. (2004). Cuestiones epistemológicas relativas al estudio psicológico de la vivencia religiosa. Psykhe, 13(1), 131-140. https://doi.org/10.4067/S0718-22282004000100011

Murray, J. D., Spadafore, J. A. \& Mcintosh, W. D. (2005). Belief in a just world and social perception: Evidence for automatic activation. The Journal of Social Psychology, 145, 35-48. https://doi.org/10.3200/SOCP.145.1.35-48

Núñez-Alarcón, M., Moreno-Jiménez, M. P. \& Moral-Toranzo, F. (2011). Modelo causal del prejuicio religioso. Anales de Psicología, 27, 852-861.

Otto, K. \& Dalbert, C. (2005). Belief in a just world and its functions for young prisoners. Journal of Research in Personality, 39, 559-573. https://doi.org/10.1016/j.jrp.2005.01.004

Paloutzian, R. F. (1996). Invitation to the psychology of religion (2a ed.). Boston, MA: Allyn \& Bacon.

Pargament, K. I. \& Hahn, J. (1986). God and the just world: Causal and coping attributions to God in health situations. Journal for the Scientific Study of Religion, 25, 193-207. https://doi.org/10.2307/1385476

Pichon, I. \& Saroglou, V. (2009). Religion and helping: Impact of target thinking styles and just-world beliefs. Archive for the Psychology of Religion, 31, 215-236. https://doi.org/10.1163/157361209X424466

Rubin, Z. \& Peplau, A. (1973). Belief in a just world and reactions to another's lot: A study of participants in the National Draft Lottery. Journal of Social Issues, 29(4), 73-93. https://doi.org/10.1111/j.1540-4560.1973.tb00104.x

Rubin, Z. \& Peplau, L. A. (1975). Who believes in a just world? Journal of Social Issues, 31(3), 65-89. https://doi.org/10.1111/j.15404560.1975.tb00997.x

Saroglou, V. (2003). Trans-cultural/religious constants vs. cross-cultural/religious differences in psychological aspects of religion. Archive for the Psychology of Religion, 25, 71-87. doi: 10.1163/157361203X00057

Saroglou, V. (2010). Religiousness as a cultural adaptation of basic traits: A five-factor model perspective. Personality and Social Psychology Review, 14, 108-125. https://doi.org/10.1177/1088868309352322

Satorra, A. \& Bentler, P. M. (2001). A scaled difference chi-square test statistic for moment structure analysis. Psychometrika, 66, 507514. https://doi.org/10.1007/BF02296192

Sibley, C. G. \& Duckitt, J. (2008). Personality and prejudice: A meta-analysis and theoretical review. Personality and Social Psychology Review, 12, 248-279. https://doi.org/10.1177/1088868308319226

Simkin, H. \& Etchezahar, E. (2013). Las orientaciones religiosas extrínseca e intrínseca: validación de la "Age Universal” I-E Scale en el contexto argentino. Psykhe, 22(1), 97-106. https://doi.org/10.7764/psykhe.22.1.477

Sutton, R. M. \& Douglas, K. M. (2005). Justice for all, or just for me? More evidence of the importance of the self-other distinction in just-world beliefs. Personality and Individual Differences, 39, 637-645. https://doi.org/10.1016/j.paid.2005.02.010

Tomaka, J. \& Blascovich, J. (1994). Effects of justice beliefs on cognitive appraisal of and subjective physiological, and behavioral responses to potential stress. Journal of Personality and Social Psychology, 67, 732-740. https://doi.org/10.1037/0022-3514.67.4.732

Watson, P. J., Sawyers, P., Morris, R. J., Carpenter, M. L., Jimenez, R. S., Jonas, K. A. \& Robinson, D. L. (2003). Reanalysis within a christian ideological surround: Relationships of intrinsic religious orientation with fundamentalism and right-wing authoritarianism. Journal of Psychology \& Theology, 31, 315-328.

Wolfradt, U. \& Dalbert, C. (2003). Personality, values and belief in a just world. Personality and Individual Differences, 35, $1911-1918$. https://doi.org/10.1016/S0191-8869(03)00040-0 
Zweigenhaft, R. L., Phillips, B. K., Adams, K. A., Morse, C. K., \& Horan, A. E. (1985). Religious preference and belief in a just world. Genetic, Social, and General Psychology Monographs, 111, 331-348.

Fecha de recepción: Octubre de 2016.

Fecha de aceptación: Junio de 2017. 